\title{
Management of acute myocardial infarction in remote and resource-limited geographies, importance of complete revascularisation, critique on renal denervation therapy for hypertension treatment, registries on DES and TAVR from different Asian countries, and much more
}

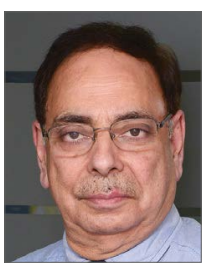

Upendra Kaul, Editor-in-Chief

The ongoing second wave of the COVID-19 pandemic in the region, with India having borne the brunt, seems to be scaling down. I am certain that we are soon going to come out of it. During the past months, we have learnt many lessons and we are getting geared up to vaccinate everybody, which is the only way to progress in this era and get back to normality in spite of new waves of the pandemic.

We just had the EuroPCR and ACC annual sessions in May and are now looking forward to the National Interventional Council (NIC) meeting of the Cardiological Society of India on the $25^{\text {th }}$ and $26^{\text {th }}$ of July. AsiaIntervention, being the official journal of this region, is launching this issue to coincide with this national conference with an introductory note by Dr Ezhilan, the Organising Secretary of the NIC.

True to its objective, this issue will focus on important issues for this region, such as the management of acute myocardial infarction and its timely treatment, which continues to be a challenge in resource-limited countries. The problems start from recognition and diagnosis to hospital arrival. Initial thrombolysis is still the most feasible method for logistic reasons. Pharmacoinvasive therapy with planned angiography and revascularisation with a hub-and-spoke model, as is already done in the Tamil Nadu STEMI programme (reviewed in this issue of the journal by Thomas Alexander, Ajit Mullasari and Brahmajee Nallamothu) is a practical method to execute this. In another study carried out in a remote region served by scanty PCI capabilities in Latin America (reported by Sameer Mehta, Carlos Campos, Roberto Botelho and colleagues in this issue), once again a hub-and-spoke model is used to recognise and manage AMI. They showed improved rates of reperfusion, resulting in a significant reduction in mortality.

When it comes to revascularisation by percutaneous coronary intervention (PCI) in patients with multivessel disease for acute coronary syndrome (ACS) or stable disease, complete revascularisation needs to be the goal. The long-term benefits of lower major adverse cardiac events (MACE) are clearly an advantage over handling only the culprit vessel. In a retrospective study with a median follow-up of 54 months reported in this issue, Praveen Goel, Ankit K. Sahu, Manas Layak et al have re-emphasised this by showing a lower rate of MACE, including improved survival, in a series of 2,960 consecutive patients from a tertiary care hospital in India. 
Two registries are reported in this issue: one is an APSIC initiative - the Asia Pacific TAVI Registry by Edgar Tay, Thet Khaing, Wei Hsian Yin and colleagues; the other is on the long-term safety and efficacy of the Resolute DES from China - the RESOLUTE China Registry by Shubin Qiao, Lianglong Chen, Shaoliang Chen et al, with a follow-up of five years. Both these registries have shown good results, comparable to those from Europe and the USA, which is indeed a matter of satisfaction for the interventional cardiologists of the region where these procedures are now well accepted by treating physicians and patients alike.

In an expert review, the subject of renal denervation therapy for hypertension is comprehensively and lucidly reviewed by C. Venkata Ram and other members of the Hypertension Council of the Cardiological Society of India. They describe the exponential rise of the therapy followed by its steep fall after the SYMPLICITY HTN-3 sham-controlled trial and then its gradual resurrection. The question to ponder is, who would benefit the most and what form of energy is preferable? That question requires an answer, and the future of renal denervation depends upon it.

In the Imaging section, Takayasu's arteritis, a rare disease in South Asian countries presenting as pulsatile neck swellings, has been shared with us by Rajesh Vijayvergiya and his colleagues, and an illustration in the form of a pseudo-pericardial effusion seen during a MitraClip procedure is presented by Ruri Ishibashi et al.

Now it is time to go through the issue with the hope that its contents will stimulate and educate our readers and promote a race to publish from the Asia Pacific region. 